\title{
Observational Evidence that Soil Moisture Variations Affect Precipitation
}

\author{
Randal D. Koster ${ }^{1}$, Max J. Suarez ${ }^{2}$, R. Wayne Higgins ${ }^{3}$, and Huug M. Van den Dool $^{3}$ \\ ${ }^{1}$ Hydrological Sciences Branch, Laboratory for Hydrospheric \\ Processes, NASA/Goddard Space Flight Center, Greenbelt, MD USA \\ ${ }^{2}$ Climate and Radiation Branch, Laboratory for Atmospheres, \\ NASA/Goddard Space Flight Center, Greenbelt, MDUSA . \\ ${ }^{3}$ Climate Prediction Center, NOAA/NWS/NCEP, Washington, D.C.
}

Submitted to Geophysical Research Letters

\begin{abstract}
Land-atmosphere feedback, by which precipitation-induced soil moisture anomalies affect subsequent precipitation, may be an important element of Earth's climate system, but its very existence has never been demonstrated conclusively at regional to continental scales. Evidence for the feedback is sought in a 50-year observational precipitation dataset covering the United States. The precipitation variance and autocorrelation fields are characterized by features that agree (in structure, though not in magnitude) with those produced by an atmospheric general circulation model (AGCM). Because the model-generated features are known to result from land-atmosphere feedback alone, the observed features are highly suggestive of the existence of feedback in nature.
\end{abstract}

\section{Introduction}

Climate scientists have long speculated that anomalous wet periods may sustain themselves through land-atmosphere feedback, by which wetter-than-normal soil from a precipitation event maintains higher-than-normal evaporation in subsequent weeks, which in turn in- 
duces additional precipitation. Similarly, through feedback, an anomalous lack of rain may induce lower evaporation rates, which in turn may reduce subsequent precipitation. Landatmosphere feedback, if it exists and is well understood, could contribute to the skill of long-term weather forecasts, including forecasts of droughts or floods.

The full feedback cycle (for convenience, discussed here in terms of wet anomalies) can be split into three parts: the wetting of the soil by precipitation, the enhancement of subsequent evaporation by the wetted soil, and the enhancement of precipitation by the evaporation. The first part is straightforward and intuitive; that it occurs in nature is indisputable. The second part, the increase of evaporation following a soil wetting, is also intuitive and is directly supported by various local evaporation meaurements (e.g., Cahill et al, 1999). It is indirectly supported by the presence of negative precipitation-temperature correlations that span much of the United States (Huang and Van den Dool [1993]), the argument being that wet soil induced by high precipitation leads to a higher surface latent heat flux at the expense of the surface sensible heat flux. The lower sensible heat flux in turn induces cooler near-surface air temperatures.

The third part of the cycle, the impact of evaporation (and thus soil moisture) on precipitation, is by far the most difficult to demonstrate with data. Observational evidence at the local scale is highly limited (e.g., Barnston and Schickedanz's (1984) study of irrigation effects), and it can be subject to contradictory interpretation (Findell and Eltahir, 1997; Salvucci et al., in press). Observational evidence at the regional to continental scale simply does not exist. Although soil wetness anomalies definitely affect precipitation in atmospheric general circulation models (AGCMs) (Shukla and Mintz, 1982; Oglesby and Erickson, 1989; Koster and Suarez, 2000; Dirmeyer et al., 2000), these studies beg an obvious question: does the simulated responsiveness of the atmosphere reflect reality, or is it just an artifact of model deficiencies?

Because of the third part of the feedback cycle, definitive proof that the full cycle occurs 
in nature is still lacking. We are limited by a paucity of long-term, spatially extensive soil moisture and evaporation data and by the difficulty of identifying causality in a highly interconnected system. (Note that isolating the impact of feedback from background noise would be especially difficult if any existing feedback is inherently weak.) Providing such definitive proof is beyond the scope of this paper. Instead, we use AGCM results in conjunction with observations to provide some new, indirect evidence that the full feedback cycle occurs in nature. The approach, reminiscent of that used by Huang and Van den Dool (1993) to study relationships between precipitation and surface temperature, is simple. Data from a pair of AGCM experiments, one in which feedback is allowed and one in which it is disabled, are compared to isolate a unique signature of feedback on the AGCM's long-term precipitation record (section 2). The signature is then sought within a recently compiled comprehensive 50-year dataset of precipitation measurements over the United States (section 3).

\section{Analysis of AGCM Precipitation}

\subsection{Structure of Correlation Fields}

Statistics from four parallel global simulations with the NASA Seasonal-to-Interannual Prediction Project AGCM, each simulation spanning the period 1948-1997, were averaged to produce the plots shown in Figure 1a. (The four simulations differed only in their initial conditions.) The first and second plots show respectively the mean (in $\mathrm{mm} \mathrm{day}{ }^{-1}$ ) and variance (in $\mathrm{mm}^{2} \mathrm{day}^{-2}$ ) of July precipitation. The third plot shows the mean, for July, of the correlations between the precipitation in one pentad (5-day period) with the precipitation two pentads later. That is, it shows the average of the correlations between precipitation amounts in 1-5 and 11-15 July, between those in 6-10 and 16-20 July, between

those in 11-15 and 21-25 July, and between those in 15-20 and 26-30 July. Presumably, if land-atmosphere feedback contributes to the prolongation of rainy periods and dry periods, then this prolongation should be reflected in the temporal correlations (and, as a result, 
in the monthly variances). We consider correlations between twice-removed pentads rather than between consecutive pentads because the latter correlations are overly influenced by storms that straddle pentads.

The AGCM runs examined in this study used a grid resolution of $2^{\circ} \times 2.5^{\circ}$. Because computed correlations depend in part on the spatial scale considered, each pentad $2^{\circ} \times 2.5^{\circ}$ precipitation field was smoothed with a three point filter in both the meridional and zonal directions prior to computing the correlations - the precipitation examined at each grid cell is actually a mix of that grid cell's precipitation and the precipitation in immediately adjacent grid cells. If the raw AGCM data were analyzed without aggregation or smoothing, an impact of soil moisture on precipitation $300 \mathrm{~km}$ away would not be picked up by the correlation calculation, even though this remote impact fully constitutes feedback. The choice of a 3-point filter (rather than either a 5-point filter or no smoothing at all) was a compromise between the desire to maximize the spatial scale considered and the desire to identify geographical variations in the structure of the correlation field. For consistency between the plots, the monthly mean and variance fields were also computed from the smoothed data. Thus, all plotted data have an intrinsic spatial scale of about $500 \mathrm{~km}$.

The patterns seen in the variance and autocorrelation plots are striking. In the variance plot, a strong maximum is seen in the center of the country. In the correlation plot, a wide swath of high correlation begins at the Gulf Coast and continues up the center of the country, eventually veering west to the Pacific Northwest. Correlations in the east and southwest are very small.

An additional 50-year simulation was performed with the AGCM in which land-atmosphere feedback was artificially disabled. In this simulation, the evaporation efficiency at the land surface was prescribed from predetermined climatological seasonal cycles, following the approach of Koster et al. (2000). Evaporation efficiency is defined here as the ratio of evaporation to potential evaporation, where potential evaporation is the maximum rate at 
which the atmosphere can receive water (as controlled by near-surface humidity gradients, wind speed, etc.). The prescription of evaporation efficiency in this simulation produced the same mean seasonal cycles of evaporation as were produced in the four control simulations without allowing wetter-than-usual soil to produce higher-than-usual evaporation rates. Sea surface temperatures varied interannually, as they did in the control simulations.

As shown in Figure 1b, this no-feedback simulation failed to produce the strong maximum of variance in the central United States, and it produced very low precipitation correlations everywhere. A comparison of Figures $1 \mathrm{a}$ and $\mathrm{b}$ provides proof that in the AGCM, the pronounced maximum of variance and the swath of high correlations seen in Figure 1a do not result from SST anomalies, internal atmospheric persistence, or other such factors they result from land-atmosphere feedback alone. In other words, at least for the AGCM's climate, these two features constitute a "unique signature" of the feedback.

\subsection{Partial Explanation of Statistical Fields}

The location of the correlation swath can be explained, at least in part, by considering the second part of the feedback cycle: the modification of evaporation by soil moisture. Here we employ a technique described by Koster and Suarez (2001). For every July in a multidecade simulation with the same modeling system, we compute the ratio $\lambda E / R_{\text {net }}$, where $E$ is monthly evaporation, $R_{\text {net }}$ is the net radiation, and $\lambda$ is the latent heat of vaporization. We then regress this ratio (a surrogate for evaporation efficiency) against the concurrent monthly soil water content (averaged over the soil column, and expressed as a degree of saturation) to obtain a slope $c$. This slope efficiently, though approximately, characterizes the sensitivity of $\lambda E / R_{\text {net }}$ to soil moisture variations. The product $c \overline{R_{\text {net }}}$, where the overline represents a climatological mean, is in turn a measure of the sensitivity of evaporation itself to soil moisture variations.

Clearly, if evaporation does not respond to a change in soil moisture (i.e., if $c \overline{R_{\text {net }}}$ is small), 
then the feedback cycle would be disrupted. A map of $c \overline{R_{\text {net }}}$, as derived from AGCM data, shows that evaporation sensitivity is indeed relatively small in both the eastern and southwestern United States, fully consistent with the low correlations seen in these two regions in Figure 1a. Indeed, the spatial pattern of $c \overline{R_{\text {net }}}$ is very similar to that of correlation in Figure 1a. Thus, variations in evaporation's sensitivity to soil moisture can explain in large part the spatial structure of the simulated precipitation correlation field.

The low sensitivities in the eastern and southwestern United States, by the way, are not unexpected. In the east, evaporation is limited by the atmosphere's ability to receive water rather than by soil water availability; evaporation there is "atmosphere-controlled". Thus, in the east, an excess or a small deficit of soil water will not affect the evaporation rate. In the southwest, evaporation sensitivity to soil moisture, particularly deeper soil moisture, which can retain anomalies from week to week, is limited by an absence of transpiration caused by sub-wilting moisture levels and minimal vegetation cover.

The third part of the feedback cycle, the modification of precipitation by evaporation, also affects the correlation fields. A map of convective available potential energy (CAPE) indicates, as expected, low values of CAPE in the southwestern United States. The low values imply a stable atmosphere that is not likely to promote convection when the nearsurface air is modified by evaporation. Thus, in the southwest, feedback and the associated temporal correlations in precipitation are further inhibited. Atmospheric stability does not similarly inhibit feedback in the central and eastern United States, where CAPE values are much higher.

As for the precipitation variance in Figure 1a, note that the monthly variance of any quantity will typically increase with both an increase in the mean and an increase in sub-monthly temporal correlation (van den Dool and Chervin, 1986). Variances in Figure 1a are large wherever the correlations are large, except where the precipitation means are low (particularly in the northwest). The result is a pronounced maximum for the variance. 
This generalization of the AGCM results is a bit of an oversimplification, since other factors also affect the shape of the AGCM's variance and correlation fields in Figure 1a. The discussion simply serves to demonstrate that, at least to first order, the structures of the simulated fields in Figure 1a are intuitively reasonable. We may indeed expect the same physical features (atmosphere control rather than soil control over evaporation, lack of transpiration, and low CAPE) to limit feedback in nature, in roughly the same regions.

\section{Analysis of Observed Precipitation}

\subsection{Description of Dataset}

The precipitation dataset used in this study is the multidecadal (1948-1997) daily precipitation reanalysis of Higgins et al. (2000). The input data for the reanalysis was a Unified Raingauge Database (URD) for the U.S., which consists of daily raingauge reports from multiple sources in the U.S., including the River Forecast Centers (about 7000 sites per day), the National Climatic Data Center (NCDC) daily cooperative network (about 6000 sites per day) and the NCDC Hourly Precipitation Network (HPN) (about 2500 sites per day; aggregated into daily accumulations). Several types of quality control were applied to the daily gauge data, including a duplicate station check, a buddy check, a standard deviation check, and a NEXRAD radar QC (see Higgins et al. 2000 for details).

The daily precipitation data were gridded at a horizontal resolution of $\frac{1}{4}^{\circ}$ latitude by $\frac{1}{4}^{\circ}$ longitude over the domain $140^{\circ} \mathrm{W}-60^{\circ} \mathrm{W}, 20^{\circ} \mathrm{N}-60^{\circ} \mathrm{N}$ using a Cressman (1959) scheme with modifications (Glahn et al. 1985; Charba et al. 1992). An intercomparison of precipitation analyses produced by Cressman (1959), Barnes (1964), Shepard (1968) and OI (Gandin, 1963) schemes revealed only minor differences in the analyses, presumably due to a sufficient data density over the U.S. (about 15500 sites per day). For full consistency with the treatment of the AGCM data, the $\frac{1}{4}^{\circ} \times \frac{1}{4}^{\circ}$ daily dataset was aggregated in space and time into a $2^{\circ} \times 2 \frac{1}{2}^{\circ}$ pentad dataset, and the horizontal fields were subsequently smoothed in 
each direction with the 3-point filter.

\subsection{Structure of the Observed Autocorrelation Fields}

Figure 1c presents the mean and variance of July precipitation and the correlation between twice-removed precipitation pentads in July, as determined from the observational dataset. The observations show that the AGCM overestimates precipitation in the eastern United States and strongly overestimates both precipitation variance and temporal correlation in the center of the country. Because the correlations are solely induced by feedback in the AGCM, we can conclude that feedback in the AGCM is excessive relative to the level of feedback in nature.

Despite these deficiencies, Figure 1c does show a pronounced maximum of precipitation variance in the center of the country, in a location very close to that predicted by the AGCM. In addition, the swath of significant correlation in July, though weaker and thinner, appears in essentially the same location as the swath in the AGCM. This co-location is highly suggestive of the existence of land-atmosphere feedback in nature, since, again, these features in the AGCM result solely from the feedback.

Other potential contributors to the observed signal are worth considering. A long-term trend in the precipitation data, for example, could lead to increases in both variance and autocorrelation. We repeated the observational analysis above using detrended precipitation data, i.e., data in which the linear temporal trend (as determined from a least-squares regression analysis) was removed from the precipitation time series at each grid cell. Results (not shown) are essentially the same as those shown in Figure 1c. Determining the relevance of still other contributors, such as monsoon dynamics, is not as straightforward; nevertheless, we can say that if these other contributors produce the observed statistical structures without help from feedback, then any agreement seen between Figures 1a and 1c is entirely coincidental. 
AGCM-generated correlations in June (not shown) are weak, and those in August are fairly strong, though not as strong as in July. (Accordingly, the product $c \overline{R_{\text {net }}}$ is generally largest in July.) Correlations inherent in the "no-feedback" simulation and in the observational data are essentially negligible in both June and August. Thus, on the one hand, the June and August results lend further support to the conclusion that the AGCM overestimates the strength of land-atmosphere feedback. On the other hand, though, we note that July is the period of maximum correlation for both the AGCM and the observations. The agreement in the timing of the maximum is either an additional piece of evidence for feedback in nature or is yet another coincidence.

\section{Discussion}

Given the potential importance of land-atmosphere feedback for improving short- and longterm weather predictions, a demonstration of the existence of feedback in nature would be of tremendous value. Although the evidence presented here is not conclusive, it is at least highly suggestive, particularly because the position of the variance and correlation structures for both the model and the observations make intuitive sense in the context of what controls feedback (section 2.2). The desired definitive evidence may need to wait several decades for the amassing of large-scale soil moisture and evaporation data. Note that the direct measurement of large-scale evaporation is a particularly difficult problem, and programs for its measurement are not currently in place. Alternatively, definitive evidence of feedback might be obtained much sooner through a detailed analysis of the impact of soil moisture initialization (as determined in a full data assimilation system) on the skill of short- or long-term precipitation forecasts.

Acknowledgments. Ping Liu helped with the processing of the AGCM data, and Guido Salvucci provided a critical reading. The NSIPP model runs were funded by the Earth Sci- 
ence Enterprise of NASA Headquarters through the EOS-Interdisciplinary Science Program and the NASA Seasonal-to-Interannual Prediction Project (NSIPP), with computational resources provided by the NASA Center for Computational Sciences.

\section{References}

Barnes, S. L., 1964: A technique for maximizing details in numerical weather map analysis. J. App. Met., 3, 396-409.

Barnston, A. G. and P. T. Schickedanz, 1984: The effect of irrigation on warm season precipitation in the southern Great Plains. J. Climate Appl. Meteor., 23, 541-554.

Cahill, A. T., M. B. Parlange, T. J. Jackson, P. O’Neill, and T. J. Schmugge, 1999: Evaporation from novegetated surfaces: surface aridity methods and passive microwave remote sensing. J. Appl. Meteor., 38, 1346-1351.

Charba, J. P., A. W. Harrell III and A. C. Lackner III, 1992: A monthly precipitation amount climatology derived from published atlas maps: Development of a digital data base. TDL Office Note 92-7, NOAA, U.S. Department of Commerce, 20 pp.

Cressman, G. P., 1959: An operational objective analysis system. Mon. Wea. Rev., 87, $367-374$.

Dirmeyer, P. A., 2000: Using a global soil wetness dataset to improve seasonal climate simulation. J. Climate, 13, 2900-2922.

Findell, K. L., and E. A. B. Eltahir, 1997: An analysis of the soil moisture-rainfall feedback, based on direct observations from Illinois. Water Resour. Res., 33, 725-735.

Gandin, L. S., 1963: Objective analysis of meteorological fields. Gidrometeor. Isdat., Leningrad. [Israel Program for Scientific Translations, Jerusalem, 1965, 242 pp.]

Glahn, H. R., T. L. Chambers, W. S. Richardson, and H. P. Perrotti, 1985: Objective map analysis for the local AFOS MOS Program. NOAA Technical Memorandum NWS TDL 75, NOAA, U. S. Department of Commerce, $34 \mathrm{pp}$.

Higgins, R. W., W. Shi and E. Yarosh, 2000: Improved United States Precipitation qual- 
ity control system and analysis. NCEP/Climate Prediction Center ATLAS No. 7, http://www.cpc.ncep.noaa.gov/research_papers/ncep_cpc_atlas/7/index.html

Huang, J. and H. M. Van den Dool, 1993: Monthly precipitation-temperature relations and temperature prediction over the United States. J. Climate, 6, 1111-1132.

Koster, R. D., and M. J. Suarez, 2001: Soil moisture memory in climate models. J. Hydromet., 2, 558-570.

Koster, R. D., M. J. Suarez, and M. Heiser, 2000: Variance and predictability of precipitation at seasonal-to-interannual timescales. J. Hydrometeorology, 1, 26-46.

Oglesby, R.J., and D.J. Erickson III, 1989: Soil moisture and the persistence of North American drought. J. Clim., 2, 1362-1380.

Salvucci, G. D., J. A. Saleem, and R. Kaufmann, Investigating soil moisture feedbacks on precipitation with tests of Granger causality, Advances in Water Resources, in press.

Shepard, D., 1968: A two-dimensional interpolation function for irregularly spaced data. ACM National Conference, 512-524.

Shukla, J., and Y. Mintz, 1982: Influence of land-surface evapotranspiration on the earth's climate. Science, 215, 1498-1501.

van den Dool, H. M. and R. M. Chervin, 1986: A comparison of month-to-month persistence of anomalies in a General Circulation Model and in the Earth's atmosphere. J. Atmos. Sci., 42, 1454-1466.

\section{Figure Captions}

Fig. 1 a. Mean precipitation (in $\mathrm{mm} \mathrm{day}^{-1}$ ), variance of precipitation (in $\mathrm{mm}^{2} \mathrm{day}^{-2}$ ), and correlations between twice-removed pentad precipitation amounts for July, as produced by the AGCM. b. Same as (a), but for an AGCM run in which landatmosphere feedback is artificially disabled. c. Same as (a), but for observational precipitation data. All correlations shown are significant at the $90 \%$ level or higher. 


\section{a. JULY PRECIPITATION, AGCM}

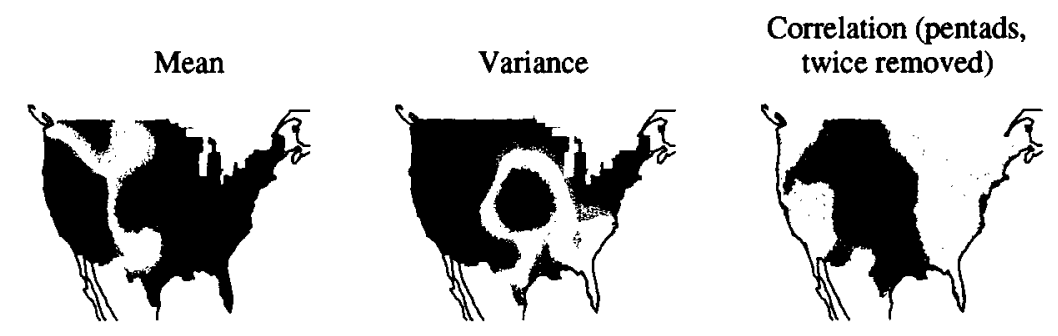

\section{b. JULY PRECIPITATION, AGCM (NO FEEDBACK)}

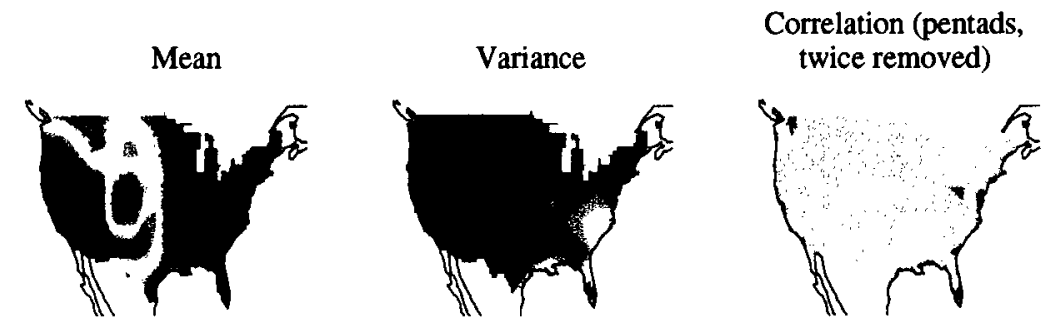

\section{c. JULY PRECIPITATION, OBSERVATIONS}
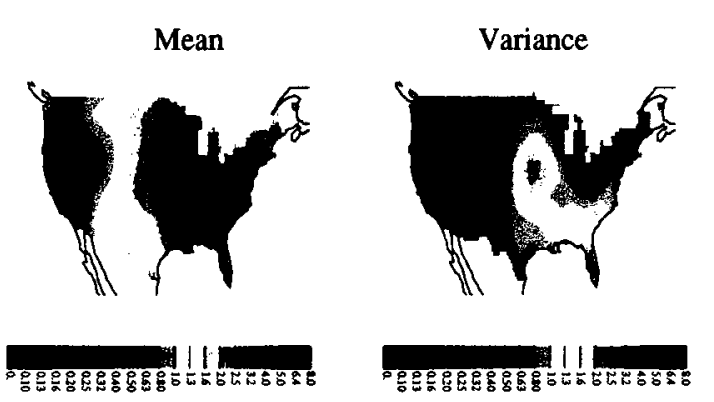

Correlation (pentads,
twice removed)
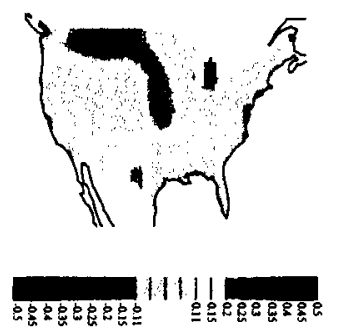

Figure 1: a. Mean precipitation (in $\mathrm{mm}^{2} \mathrm{day}^{-2}$ ), variance of monthly precipitation (in $\mathrm{mm}^{2} \mathrm{day}^{-2}$ ), and correlations between twice-removed pentad precipitation amounts for July, as produced by the AGCM. b. Same as (a), but for an AGCM run in which land-atmosphere feedback is artificially disabled. c. Same as (a), but for observational precipitation data. All correlations shown are significant at the $90 \%$ level or higher. 
"Observational Evidence that Soil Moisture Variations Affect Precipitation", by R. Koster, M. Suarez, W. Higgins, and H. Van den Dool

\section{Statement of Significance}

QUESTION: Although hydrologists and climatologists often assume that precipitation-induced soil moisture anomalies can infuence subsequent precipitation, they have never been able to prove this on the large scale with observational data. Although direct evidence is indeed impossible to obtain, can we find some indirect evidence in the available observations?

APPROACH: Two simulations were performed with an atmospheric general circulation model: one that allowed soil moisture to affect precipitation, and one that did not. A comparison of the two simulations identified some unique signatures of soil moisture impact in the simulated precipitation data. The same signatures were sought in a 50-year observational precipitation dataset covering the United States.

SIGNIFICANCE AND IMPLICATIONS OF FINDINGS: The "soil moisture impact" signatures are not as strong in the observational data as they are in the model results, suggesting that the model overestimates the soil moisture precipitation connection. Nevertheless, the signatures do appear in the observations. Thus, we have found, for the first time ever, indirect large-scale evidence supporting the idea that soil moisture variations do influence precipitation in the real worki.

\section{RELATIONSHIP TO ESE SCIENCE PLAN}

The soil moisture - precipitation connection is key to many science investigations that address ESE themes of (1) seasonal-to-interannual climate prediction, (2) changes in long term climate, and (3) landcover and land use change. 


\section{Popular Summary:}

"Observational Evidence that Soil Moisture Variations Affect Precipitation", by R. Koster, M. Suarez, W. Higgins, and H. Van den Dool

Climate scientists have long speculated that wet periods may sustain themselves through land-atmosphere feedback, by which wetter-than-normal soil from a precipitation event maintains higher-than-normal evaporation in subsequent weeks, which in turn induces additional precipitation. Similarly, through feedback, an anomalous lack of rain may induce lower evaporation rates, which in turn may reduce subsequent precipitation. Land-atmosphere feedback, if it exists and is well understood, could contribute to the skill of long-term weather forecasts, including forecasts of droughts or floods.

Important as it is, the existence of this feedback has never been demonstrated conclusively with observational data, largely due to a lack of large-scale soil moisture and evaporation measurements that span decades. Any evidence of feedback must be indirect and circumstantial. In this paper we search for such indirect evidence in a 50-year observational precipitation dataset covering the United States.

The approach involves the use of an atmospheric general circulation model. Two simulations were performed with the AGCM: one that allowed soil moisture to affect precipitation, and one that did not. By comparing the precipitation fields generated by the two simulations, we could identify some unique signatures of land-atmophere feedback. These same signatures were then sought in the observational precipitation dataset.

The feedback signatures were not as strong in the observational data as they were in the model results, suggesting that the model overestimates the soil moisture - precipitation connection. Nevertheless, the signatures did appear in the observations. Thus, for the first time ever, we have indirect large-scale evidence that supports the existence of land-atmosphere feedback, evidence that supports the idea that soil moisture variations do influence precipitation in the real world. 